Conclusions This study provide strong evidence that MSCs implantation ameliorates interstitial fibrosis and the remodelling of gap junction and Kv4.2 expression, attenuates focal heterogeneity of reporlarisation and conduction and reduces vulnerability to VTs. These results suggest that MSC transplantation might be emerge as a new preventive strategy against VAs besides improving cardiac performance in ischaemic heart disease.

\section{e0227 INTRACORONARY INFUSION OF MESENCHYMAL STEM CELLS REDUCES PROARRHYTHMOGENIC RISKS IN SWINE WITH MYOCARDIAL INFARCTION}

doi:10.1136/hrt.2010.208967.227

Wang Deguo, Jin Yan, Ding Chandong, Zhang Fengxiang, Zhang Minglong, Yang Bing, Shan Oijun, Zou Jiangang, Cao Kejiang. The First Affiliated Hospital of Nanjing Medical University, Nanjing, Jiangsu, China

Objective To evaluate the risk of ventricular arrhythmias (VAs) after MSC transplantation in swine model with acute myocardial infarction.

Methods Swine models with myocardial infarction were created by intracoronary balloon occlusion and then received MSC solution or $0.9 \%$ sodium chloride solution via balloon catheter. 6 weeks after artery occlusion, heart rate turbulence (HRT), dispersion of APD and RT (APDd and RTd), slope of APD reconstitution curve, Threshold cycle length of APD alternan and cardiac electrophysiologic study (EPS) were used to evaluated the VAs risks. Haemodynamic study was assessed to evaluate the cardiac performances. The concentrations of collagen in non-infarcted myocardium was assayed to elucidate the degree of myocardial remodelling.

Results There were significantly abnormality of turbulence onset (TO) and turbulence slope (TS) in MI group relative to control group $(p<0.01)$. MSC transplantation could ameliorate the abnormal HRT (MSC group vs MI group, $p<0.01$ ). The values of APD90, APDd, RT and RTd in the MI and MSC group markedly increased compared with the control group $(p<0.01)$. These parameters in the MSC group were significantly lower than MI group $(\mathrm{p}<0.05)$. The slope of reconstitution curve in the MSC group was higher than control group but lower than MI group. The threshold cycle length of APD alternan in the MSC group was remarkably higher than that in the control group $(p<0.01)$ and lower than that in the MI group $(\mathrm{p}<0.05)$. Inducible malignant VAs in the MSC group were remarkable lower than that in the MI group (30.8\% vs $70.0 \%)$. MSCs therapy markedly improve impaired cardiac performances and reduce fibrosis deposition after MI.

Conclusions MSC intracoronary infusion does not cause proarrythmogrenic risk but tent to reduce the risk of malignant VAs. MSC therapy might be emerge as a new, safe and effective preventive strategy against VAs besides improving cardiac performance in ischaemic heart disease.

\section{e0228 TRANSFECTION OF RECOMBINANT ADENO-ASSOCIATED VIRUS SEROTYPE 9 TO MOUSE HEART IN VIVO AND THE EFFECTS ON CARDIAC FUNCTION}

doi:10.1136/hrt.2010.208967.228

Xiang Yiang, Ma Yitong, Yang Yining, Chen Bangdang, Liu Fen, Gao Xia. Department of Cardiovascular Medicine, The First Affiliated Hospital Xinjiang Medical University, China

Objective To evaluate the transfection efficiency of recombinant adeno- associated virus serotype 9 carrying enhanced green fluorescent protein (rAAV9- eGFP) to mouse heart in vivo and the effects on cardiac function.

Methods 1.16 C57BL/ 6 mice were transfected rAAV9-eGFP by tail injection. EGFP expression in the heart, liver, lung, kidney and brain cryosections was observed under inverted fluorescence microscope 7 , 14, 21, 28 days after the injection of rAAV9-eGFP and eGFP was quantitated by Western Blot. 2. 20 C57BL/ 6 mice were divided into control group and rAAV9-eGFP group randomly, and were received with saline or rAAV9-eGFP. The echocardiography and haemodynamics were performed 28 days after the injection of saline or rAAV9-EGFP.

Results 1. EGFP expression in the heart reached the maximum at day 21 , at the point of which the transduction efficiency of rAAV9eGFP in myocardium was $32 \%$. The other tissues had a little or no eGFP expression. 2. The cardiac function did not reveal significant difference between rAAV9-eGFP group and the control group after transfection ( $p>0.05)$.

Conclusion rAAV9-eGFP gene can be stably and efficiently expressed in mouse heart, and has no toxic effect on cardiac function.

\section{e0229 INHIBITION OF NF-KB ATTENUATES POST-INFARCT LEFT VENTRICULAR RUPTURE AND REMODELLING IN AGED MICE BY RIBOZYME GENE TRANSFER WITH ADENO-ASSOCIATED VIRUS SEROTYPES 9}

doi:10.1136/hrt.2010.208967.229

Xiang Yang, Ma Yitong, Yang Yining, Chen Bangdang, Liu Fen, Gao Xia, Du Lei. Department of Cardiovascular Medicine, The First Affiliated Hospital Xinjiang Medical University, Urumqi, China

Objective Using intravenous injection of adeno-associated virus serotypes 9 carring ribozyme gene (AAV9-R65), we examined whether inhibition NF- $\kappa B$ would prevent post-infarct left ventricular rupture and remodelling in aged mice.

Methods and results Old (18-month-old) C57BL/6 male mice were given AAV9-R65 by tail vein injection 16 days before operation (MI+R65). Myocardial infarction was induced by ligation of the left coronary artery in MI+R65 group and myocardial infarction (MI) group mice. NF- $\mathrm{KB}$ activity was inhibited in MI+R65 mice. Inhibition of NF- $\kappa \mathrm{B}$ reduced cardiac rupture in $\mathrm{MI}+\mathrm{R} 65$ group $(15.2 \%$ vs $32.8 \%, p=0.018)$. Echocardiographic measurements revealed that diameter of LV was significantly decreased, and ventricular wall thickness, fraction shortening were significantly increased in MI+R65 mice compared with MI mice $(p<0.05)$. MMP-9 and TNF- $\alpha$ were decreased in MI+R65 group $(p<0.05)$. And collagen was also decreased in MI+R65 group $(p<0.05)$. But there were no changes of IL-1 $\beta$ in MI+R65 group.

Conclusions Cardiac rupture and remodelling were attenuated in aged mice by ribozyme gene transfer with adeno-associated virus serotypes 9 . It maybe caused by decreased collagen as the result of decreased MMP-9, TNF- $\alpha$ which proved that NF- $\kappa$ B signal pathway may be associated with cardiac rupture and remodelling in aged mice.

\section{e0230 THE EFFECT OF CO-CULTURING WITH NATIVE CARDIOMYOCYTES ON ASCORBIC ACID-INDUCED CARDIOMYOGENIC DIFFERENTIATION IN EMBRYONIC STEM CELLS}

doi:10.1136/hrt.2010.208967.230

Ou Dongbo, He Yong, Wang Zikuan, Liu Xiongtao, Lee Jun, Wang Hongtao, Guo Wangang, Zheng Oiangsun. Department of Cardiology Tangdu Hospital Fourth Military Medical University, Xi'an, China

Objective Ascorbic acid has been reported to promote the differentiation of embryonic stem cells (ESCs) into cardiomyocytes (CMs). However, appropriate culture protocols are needed to improve the differentiation efficiency and produce adequate 
numbers of CMs for therapeutic cell transplantation. This study investigated the effect of co-culturing with native CMs on ascorbic acid-induced cardiomyogenic differentiation in embryonic stem cells, to develop a novel protocol for generating functional CMs from ESCs.

Methods Native CMs were isolated from the hearts of 1-day-old Sprague-Dawley rats. Mouse ESCs were cultured in hanging drops to form embryoid bodies (EBs) and treated with or without $0.1 \mathrm{mM}$ of ascorbic acid (Sigma) for cardiomyogenic differentiation. They were divided into four groups: ascorbic acid \& co-culture group, co-culture group, ascorbic acid group, and control group. In the coculture system, EBs were co-cultured with native CMs by the hanging cell culture inserts (PET $1 \mu \mathrm{m}$ ) (Millicell; Millipore, Bedford, MA, USA). The native CMs were purposely placed on culture plate inserts to prevent direct contact with subnatant EBs. Both the ESCs and native CMs grew in the same medium but they were easy to separate. The structural and functional properties of ESC-derived CMs (ESCM) were evaluated by microscopic observation, immunocytochemistry, RT-PCR, and transmission electron microscopy.

Results The average percentages of EBs exhibited rhythmic contractions in co-culture and ascorbic acid group, co-culture group, ascorbic acid group, and control group were $86.6 \pm 9.52 \%, 65.60 \pm 10.77 \%$, $29.6 \pm 6.03 \%$, and $17.76 \pm 5.99 \%$, respectively. The percentage of beating EBs in co-culture \& ascorbic acid group was much higher and the homogeneity of EBs were significantly improved over that seen in other groups $(p<0.01)$, simultaneously, the automaticity of beating also maintained for more time. The majority (>90\%) of cells in EBs were ESCM that acquired almost the same structural and functional properties as typical CMs.

Conclusions The present study demonstrates the cardiomyogenic differentiation of ESCs can be efficiently controlled by co-cultured with native CMs, and this may lead to a practicable cocktail approach to generate ESC-derived CMs for stem cell-based regenerative medicine.

\section{e0231 POLYMER-FREE SIROLIMUS AND PROBUCOL-ELUTING STENT FOR RENAL ARTERY: AN INITIAL EXPERIENCE IN SWINE}

doi:10.1136/hrt.2010.208967.231

Zheng Bin, Yan Hongbing. Beijing Anzhen Hospital, Beijing, China

Objective Bare metal stent (BMS) implantation can resolve renal artery stenosis successfully, but in-stent restenosis does occur, especially in small diameter renal arteries $(<5.0 \mathrm{~mm})$. The aim of this study is to test whether a newly designed polymer-free sirolimus and probucol-eluting stent (SPES) can inhibit neointimal hyperplasia of renal artery in swine.

Methods 26 stents (18 SPES and 18 BMS) were implanted in 36 renal arteries of 18 animals. During every procedure, a SPES and a BMS were randomised to the right or left renal artery. Seven animals were sacrificed after 90 days, and 11 after 180 days.

Results Histomorphometric analysis was performed. After 90 days, minimal lumen area, neointimal area, score of inflammation and score of endothelialisation were not significantly different between BMS and SPES. After 180 days, minimal lumen area was not significantly different between BMS and SPES $\left(6.55 \pm 2.91 \mathrm{~mm}^{2}\right.$ vs $\left.7.32 \pm 1.99 \mathrm{~mm}^{2}, \mathrm{p}=0.477\right)$, but neointimal area was significantly less in SPES vessels than in BMS vessels $\left(3.07 \pm 0.83 \mathrm{~mm}^{2}\right.$ vs $\left.4.47 \pm 1.23 \mathrm{~mm}^{2}, p=0.005\right)$. Score of inflammation and score of endothelialisation were not significantly different between BMS and SPES after 180 days.

Conclusion SPES can successfully inhibit neointimal hyperplasia of renal artery in swine. At the same time, inflammation and endothelialisation in SPES vessels were similar to BMS vessels.

\section{e0232 THE CHANGE OF VENTRICULAR INA AT DIFFERENT TIME OF SIMULATED ISCHAEMIA AND THE EFFECT OF ATORVASTATIN}

doi:10.1136/hrt.2010.208967.232

Li Hongshi, Bian Bo, Wan Zheng, Teng Tianming, Wang Fang. Tianjin Medical University General Hospital, Tianjin, China

Objective To observe time dependent effects of simulated ischaemia on transient sodium currents $\left(\mathrm{I}_{\mathrm{Na}}\right)$ of rat left ventricular myocytes, and the effects of atorvastatin on ischaemia $\mathrm{I}_{\mathrm{Na}}$.

Methods 30 Wistar rats were used for isolating left ventricular myocytes, which were randomly divided into two groups: ischaemia group (normal $\rightarrow$ simulated ischaemia) and statin group (normal $\rightarrow$ simulated ischaemia with $5 \mu \mathrm{mol} / 1$ atorvastatin). $\mathrm{I}_{\mathrm{Na}}$ were recorded in normal condition (for control) by whole-cell patchclamp. Then in simulated ischaemia condition, $\mathrm{I}_{\mathrm{Na}}$ were recorded from 3 to $21 \mathrm{~min}$, monitored normalised peak currents every $2 \mathrm{~min}$, and compared gate parameters between normal and simulated ischaemia ( $3 \mathrm{~min}$ ) condition.

Results a. Normalised currents (at $-40 \mathrm{mV}$ ), in ischaemia group, compared with normal $(0.95 \pm 0.04)$, the currents in simulated ischaemia were increased to peak at $3 \mathrm{~min}(1.15 \pm 0.08, \mathrm{p} 0.05$, respectively), and decreased at $21 \mathrm{~min}(0.56 \pm 0.13, \mathrm{p} 0.05)$. b. Gate parameters, from normal to simulated ischaemia condition at $3 \mathrm{~min}$, membrane potential at $50 \%$ maximal activation $\left(\mathrm{V}_{1 / 2, \mathrm{a}}\right)$, offsetting of activation curve $\left(\mathrm{K}_{\mathrm{a}}\right)$, membrane potential at $50 \%$ maximal inactivation $\left(\mathrm{V}_{1 / 2, \mathrm{i}}\right)$ and deinactivation constant $(\tau)$ were decreased $(\mathrm{p}<0.01$, respectively) in ischaemia group, but offsetting of inactivation curve $\left(\mathrm{K}_{\mathrm{i}}\right)$ were not changed; compared between two groups, $K_{i}$ of statin group were decreased $(p<0.05)$ and the decrease of $\tau$ value in statin group were less than ischaemia group $(p<0.05)$.

Conclusions The effects of simulated ischaemia on $\mathrm{I}_{\mathrm{Na}}$ are time dependent, while $\mathrm{I}_{\mathrm{Na}}$ is transient increased at $3 \mathrm{~min}$, and atorvastatin can depress this process.

\section{e0233 LATE GADOLINIUM-ENHANCED MRI IN RESTRICTIVE CARDIOMYOPATHY}

doi:10.1136/hrt.2010.208967.233

Zhao Shihua, Jiang Shiliang, Cheng Huaibing, Lu Minjie, Ling Jian, Zhan Yan, Yan Chaowu, Liu Oiong, Ma Ning, Li Shiguo, Yin Gang. Department of Radiology, Cardiovascular Institute and Fuwai Hospital, Chinese Academy of Medical Sciences, Peking Union Medical College, Beijing, China

Objective To evaluate the diagnostic value of MRI in combination of late gadolinium-enhanced imaging in the identification of restrictive cardiomyopathy (RCM).

Methods 116 patients with RCM underwent ECG, x-ray, Echocardiography and MRI. The final diagnosis was made on comprehensive evaluation in consideration of patients' history, clinical symptom and sign, imaging modalities. All patients had objective evidence of impaired cardiac filling and were referred to rule out pericardial thickening. Five histologically proven cases with RCM included heart transplantation in four patients with RCM, endomyocardial biopsy in one patient with RCM. Fifty-five normal subjects were used for reference. All patients were divided into two groups according to contrast-enhanced MRI: RCM with delayed enhancement (RCM with DE, $n=35$ ) and RCM without delayed enhancement (RCM without DE, $n=81$ ). Quantitative measurement of bi-atrial and bi-ventricular size, ventricular septal and left free wall thickness were done. A paired t-test was used for statistic analysis and a $p$ value of less than 0.05 was considered significant. Qualitative assessment of segmental wall motion, in this present study.

Results The parameters, such as bi-atrial size, right ventricular diastolic diameter (RVDD), ventricular septal and left free wall 